\title{
Generation of the desired arrays of a perfect vortex beam
}

\author{
Z Behjat and A Sabatyan \\ Department of Physics, Science Faculty, Urmia University, Urmia, Iran
}

E-mail: a.sabatyan@urmia.ac.ir

(Received 16 March 2019 ; in final form 03 September 2019)

\begin{abstract}
In this paper, we introduce a novel diffraction element for generating any desired arrays of the vortex and perfect vortex beam. The method is based on combining the radially phase shifted spiral zone plate with different gratings. We show that the element has a great potential in generating a variety of arrays with the desired vortex ring radius and topological charges. We can assemble various vortex and perfect vortex beams not only in a lattice array, but also in a tilted lattice or circular arrays. Reported vortex arrays are in the group of vortices having the same topological charge $p$, so the total topological charge of MP, where M is the number of elements. The experimental results are in a good agreement with the simulation predictions.
\end{abstract}

Keywords: diffraction, Fresnel zone plate, vortex beam, perfect vortex beam

For full article, refer to the Persian section 